Damaris Nübling (Mainz)

\title{
Sprachverfall? Sprachliche Evolution am Beispiel des diachronen Funktionszuwachses des Apostrophs im Deutschen
}

\begin{abstract}
In der emotional geführten Sprachverfallsdebatte wird besonders die Apostrophsetzung vor dem Genitiv- und dem Plural-s, vulgo Deppen-Apostroph, kritisiert und als vermeintliche Entlehnung aus dem Englischen stigmatisiert. Erst seit kurzem liegen mit Scherer (2010, 2013) korpusbasierte Untersuchungen vor, die eine angemessene Interpretation dieses graphematischen Wandels erlauben, der weitaus älter ist als gemeinhin vermutet. Generell erweist sich, dass viele als neu und bedrohlich empfundene Sprachveränderungen bereits vor über hundert Jahren meist ebenso emotional gegeißelt wurden. Der Beitrag befasst sich hauptsächlich mit der diachronen Entwicklung des phonographischen Apostrophs zu einem morphographischen, dessen Funktion nun nicht mehr darin besteht, nicht-artikulierte Laute zu markieren, sondern morphologische Grenzen (Uschi's, Joseph K.'s, CD's). Deutlich wird, dass der Apostroph der Gestaltschonung komplexer Basen dient, deren Gros aus Eigennamen besteht. Anschließend wird in einem kürzeren Teil nach der Entstehung und Beschaffenheit dieser $s$-Flexive selbst gefragt. Diese sind ihrerseits Ergebnis flexionsmorphologischer Umstrukturierungen und garantieren maximale Konstanthaltung des Wortkörpers. Abschließend wird noch die neueste Entwicklung gestreift, die in der Deflexion ebendieser $s$-Flexive besteht und die sich wieder am deutlichsten bei den Eigennamen manifestiert. Diese haben als Quelle all dieser Entwicklungen zu gelten (vgl. des Irak, des Helmut Kobl, auch des Perfekt, des LKW, des Gegenüber). Insgesamt ist festzustellen: Nicht nur die Apostrophsetzung vor $s$-Flexiven, sondern auch die $s$-Flexive selbst sowie ihr derzeitiger Abbau dienen ein und derselben Funktion: Der Schonung durch Konstanthaltung markierter Wortkörper, worunter mehrheitlich Eigennamen fallen, daneben auch Fremdwörter, Kurzwörter und Konversionen. Damit sind es die Eigennamen, die Ausgangspunkt und Ursache tiefgreifenden flexionsmorphologischen und graphematischen Wandels bilden.
\end{abstract}

\section{Das Deutsche als tiefes Schriftsystem}

Das deutsche Schriftsystem hat seit der frühnhd. Periode eine rapide zunehmende Vertiefung seiner Verschriftungsprinzipien erfahren, d.h., es hat neben bislang phonographischen auch morphosemantische Prinzipien ausgebildet. Am wichtigsten ist dabei das so genannte Morphemkonstanzprinzip. Es besagt nach Nerius (2000, S. 146 f.), dass

graphische Morphemformen - z.T. trotz Abweichung in der phonischen Morphemform - weitgehend konstant oder zumindest ähnlich gehalten werden, um eine Identifizierung der Morpheme durch den Lesenden zu befördern. Von daher erklären sich auch konkurrierende Bezeichnungen wie Prinzip der Morphemkonstanz. oder Schemakonstan\%: 
Das Morphemkonstanzprinzip ist vielgestaltig und in seiner Herausbildung eingehend von Ruge (2004) untersucht worden. Der Kurzvokal $[\varepsilon]$ und der Diphthong [0i] können jeweils auf zwei Weisen verschriftet werden, entweder phonographisch als $<$ e $>$ bzw. $<$ eu $>$ (z.B. $<$ Stelle $>$, $<$ heute $>$ ) oder morphographisch mit den Umlautgraphemen $<$ ä $>$ bzw. $<a ̈ u>~(z . B .<$ Ställe $>$, $<$ Häute $>$ ). Letztere stellen einen materiell-graphetischen Bezug zu nichtumgelauteten Basisformen mit $<\mathrm{a}>$ bzw. $<$ au $>$ her $(<$ Stall $>$ bzw. $<$ Haut $>)$. Der faktisch (auf phonologischer Ebene) große Abstand zwischen [a] und $[\varepsilon]$ bzw. [au] und [oi] wird auf graphematischer Ebene durch die größere Ähnlichkeit der Buchstaben verringert (als $<a / a ̈>b z w .<a u / a ̈ u>)$. Dies fördert die direkte Informationsentnahme durch den (stillen) Leser (Erfassungsfunktion). Die morphologischen $<\ddot{a} / \ddot{a} u>$-Schreibungen kommen um 1500 auf und setzen sich zügig zwischen 1600 und 1700 durch.

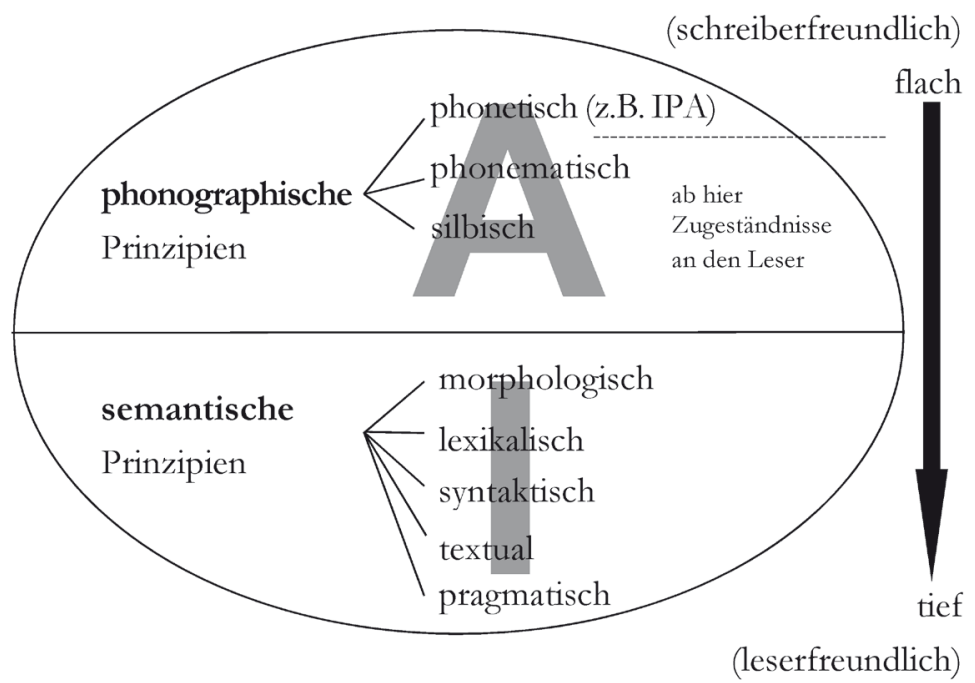

Abb. 1: Vom flachen zum tiefen Schriftsystem

Weitere von Ruge (2004) untersuchte morphologische Prinzipien bestehen in der Übernahme der Konsonantendoppelschreibung von der Wortmitte (wollen, können) in den Silbenendrand (<will, wollte, kannst>). Ursprünglich diente diese Graphemverdopplung nur (phonographisch) der intervokalischen Silbengelenkschreibung in <wollen, sollen, können $>$. Sie wurde morphologisch überformt und in sämtliche Wortformen überführt $(<$ wil $\rightarrow$ will, solte $\rightarrow$ sollte $>$ ), womit morphologische Schema- oder Konstanzschreibung entstand. Als drittes hat Ruge die graphische Aufhebung der Auslautverhärtung untersucht $(<\mathrm{tac}>\rightarrow<$ Tag $>$ [ta:k] wegen $<$ Tage $>$; schon gegen 1500 durchgeführt). Viertens, und dies leitet zu den Apostrophen über, fand ein „Abbau determinativ-phonetischer Kürzungen“ (ebd., S. 207-232) statt, d.h., 
schreiberbegünstigende Kürzungszeichen (in Handschriften ermöglichten sie u.a. auch die Einhaltung des Schriftspiegels), die nicht immer eindeutig waren, mussten vom Leser aufgelöst werden. So konnte der Nasalstrich, angebracht über dem vorangehenden Vokal, sowohl $<$ m $>$ und $<$ mm $>$ als auch $<\mathrm{n}>$ und $<\mathrm{nn}>$ ersetzen. Der Leser hatte so zu ergänzen, dass sich etwas Sinnvolles ergab. Dies gilt auch für den so genannten $(e) r$-Haken, der für $<\mathrm{r}>$ und (i.d.R.) einen Vokal stand, oft $<$ er $>$, und meist rechts am vorangehenden Graphem angebracht wurde. Solche Kürzungszeichen unterminieren zum einen die Morphemkonstanz (indem sie Schreibvarianten wie <ganz> vs. $<$ gāz $>$ generieren), zum anderen muten sie dem Leser die je spezifische Auflösung zu. So nimmt es nicht wunder, dass die Kürzungszeichen zwischen 1500 und 1600 nicht nur rapide zurückgehen, sondern regelrecht abstürzen und bald danach verschwinden (siehe die Graphik in Ruge 2004, S. 217).

Jacob Grimm wendet sich in seiner polemischen Schrift „Ueber das pedantische in der deutschen sprache" von 1847 an den Stellen, wo er die deutsche Schreibung behandelt, fast ausschließlich gegen solche (und andere) morphosemantische Prinzipien, deren Funktionalität damals noch nicht bekannt war. Stattdessen möchte er zur flachen Phonographie des Ahd. und Mhd. zurückkehren, wenn er schreibt:

vor mehr als 800 jahren, zu Notkers zeiten in Sanct Gallen, war es besser um die deutsche schreibung bestellt und auf das genaue bezeichnen unsrer laute wurde damals grosze sorgfalt gewendet; noch in der schrift des 12. und 13. jh. läszt sich rühmliches melden, erst seit dem 14. jh. begann sie zu verwildern. mich schmerzt es tief gefunden zu haben, dasz kein volk unter allen [...] heute seine sprache so barbarisch schreibt wie das deutsche [...].

Zu den Barbareien zählt er die Schreibung einfacher Laute mit mehr als einem Buchstaben (z.B. $[\chi] \rightarrow<\mathrm{ch}>$, [J] $\rightarrow<$ sch $>$ ), was er als ,schleppende Breite" empfindet, die Verdreifachung von Graphemen in der Morphemfuge, obwohl man sie nur einfach artikuliert (<stallicht, betttuch $>$ ), die unnötige Schreibung < wangküsse >, wo - er argumentiert stets aus phonographischer Perspektive - ja auch die mhd. Schreibung < wanküsse $>$ ausreiche (vor $[\mathrm{k}]$ wird $[\mathrm{n}]>[\mathrm{n}]$ assimiliert). Auch Bindestriche innerhalb von Komposita (<himmel-blau $>$ ) und Apostrophe (als Fälle „,unnöthiger striche und haken"), die auch Grimm schon als Import aus dem Französischen bzw. Englischen abwertet, seien unnötig, da sie keine Laute bezeichnen. Die größten Barbareien stellen für ihn jedoch die Eigennamenschreibung und die Substantivgroßschreibung dar. Zu ersterer: „Jeder regel des schreibens aber enthoben wähnt man sich sonst bei eigennamen", denn

man schlage eins unsrer adreszbücher auf, welche barbarei daraus entgegen weht; da stehn Hofmänner und Wölfe bald mit F bald FF geschrieben [...]; unerläszlich erscheint mir, dasz eine gebildete sprache ihre eigennamen den gesetzen unterwerfe, die für alle übrigen wörter gelten, und wo sie es nicht thut verdient sie geschmacklos zu heiszen. 
Eigennamen befinden sich bis heute jenseits orthographischer Regulierung, was ihrer besonderen Funktion geschuldet ist, monoreferent auf ein Objekt zu verweisen, ohne die potenzielle Semantik, die ihr Wortkörper evozieren könnte, zu entfalten (z.B. *,Wolf' in $<$ Wolff $>$ ). Diese wörtliche Bedeutung wird ausgeblendet. Abweichende Schreibungen unterstützen diese Funktion. ${ }^{1}$ Außerdem verleihen sie dem Eigennamen maximale Unverwechselbarkeit und Individualität. Genaugenommen kommen die deutschen Eigennamenschreibungen dem logographischen Prinzip am nächsten, d.h. der individuellen Wortschreibung, oft bei Verletzung der üblichen GPK-Regeln in beide Richtungen (z.B. Konsonantenverdopplungen nach Liquidgraphemen wie in $<$ Wolff, Bismarck $>$ oder kurze Vokalartikulation bei $<$ Hofmann, Schmid $>$ ).

Da auch die Substantivgroßschreibung dem Mhd. fremd war, hat Grimm diesen „misbrauch groszer buchstaben“ strikt abgelehnt. Auch die Großschreibung fällt unter die morphosemantischen Prinzipien, am ehesten unter das syntaktische Prinzip, indem Köpfe von NPs markiert werden. Zu weiteren Verschriftungsprinzipien siehe Augst (1981), Meisenburg (1998), Eisenberg (1989), Nerius (2007).

\section{Die Entwicklung des Apostrophs vom phonographischen zum morphographischen Syngraphem}

\subsection{Historische Entwicklung}

Der Apostroph hat eine interessante Entwicklung durchlaufen und sich dabei, ganz im Einklang mit der allgemeinen Überwindung der reinen Phonographie hin zu einem tiefen Schriftsystem, von einem phonographischen zu einem morphographischen Zeichen gewandelt. Da er am vorangehenden Graphem als „Hinter-“ oder „Nachstrichlein“ (so seine Bezeichnung im 17. Jahrhundert) haftet und sich auf Wortformen und deren Interpretation bezieht (und nicht auf die Syntax), bezeichnet man ihn auch als Syngraphem (Gallmann 1989). Die folgenden Ausführungen basieren auf Klein (2002), Ewald (2006), Nerius (2007) und Bankhardt (2010).

In mittelalterlichen Handschriften fungierte der Apostroph oder $(e) r$ Haken, wie bereits erwähnt, als rein graphisches Kürzelzeichen für zu arti-

Bei (oft komplexen) Namen, die aus dem Normalwortschatz schöpfen, z.B. solchen von Werktiteln (,Auf der Suche nach der verlorenen Zeit“, „Die Räuber“, „Der Fliegende Holländer"), findet üblicherweise eine Rahmung durch Anführungszeichen statt. Diese Anführungszeichen können gewissermaßen als doppelte Apostrophe gesehen werden. In jedem Fall markieren sie den onymischen Status ihres Inhalts und blockieren gleichzeitig die Aktivierung der wörtlichen Bedeutung. Sie bilden einen noch markanteren Eigennamenschutz als der Apostroph (auch durch ihre Paarigkeit) und können als echte logographische Indikatoren gewertet werden. 
kulierendes $<\mathrm{r}>$ (nach Vokal, z.B. $<\mathrm{ja}{ }^{\prime}>$ ) oder $<\mathrm{er}>$ (dies nach Konsonant, z.B. <od', v'gessen, kind'> (Ebert et al. 1993, S. 31). Auch machen Ebert et al. (1993, S. 32) ab dem 16. Jahrhundert französischen Einfluss geltend, was seinen Elisionswert für $\langle\mathrm{e}\rangle$ erklären würde. Auch wenn er keinen eindeutigen Laut bezeichnete, so war der Apostroph anfänglich durchaus ein Phonogramm: Er stand für einen Laut/eine Lautfolge, der/die beim Lesen des Textes auszusprechen war und nur auf graphematischer Ebene nicht realisiert wurde. Wie von Ruge (2004, S. 207-232) gezeigt, wird dieses Kürzel in den frühnhd. Drucken zügig abgebaut. Damit wird das Zeichen in gewisser Weise frei. Es existiert Form ohne (bzw. mit schwindender) Funktion - beste Voraussetzung für eine Reanalyse. Von hier aus wandelt sich der Apostroph zu einem Elisions- oder Auslassungszeichen, meist für $\langle\mathrm{e}\rangle$, das im Gegensatz zur vorherigen Funktion genau nicht artikuliert wird. Es sind die metrischen Anforderungen der Dichtersprache des 17. Jahrhunderts, in denen sich eine solche Funktion entfalten konnte. Opitz und Schottelius haben den Apostroph in dieser Funktion ausführlich beschrieben (Klein 2002, S. 184). Primär bezeichnete er Apokopen (solch' augen) und Synkopen (Gott's Verstand), später (im 18. Jahrhundert) auch Aphäresen. Im 17. Jahrhundert entsteht die Diskussion um einen Standard, an dem die damalige Literatursprache großen Anteil hatte. In diesem Spannungsfeld zwischen apo- und synkopierender Volkssprache bzw. Dialekten und einer Literatursprache mit vollen Formen wurde der Apostroph mit der Konnotation ,nicht standardsprachlich', ,volkssprachlich', ,gesprochensprachlich befrachtet. Damit drückte er eine Differenz aus, die auch als Defizit empfunden wurde. Der Apostroph erlaubt sozusagen nicht-standardkonformen Wörtern den Zutritt in die Schriftsprache und markiert sie gleichzeitig als eigentlich nicht befugt. Der Drang zu sprachlicher De- bzw. Überregionalisierung, d.h. Vereinheitlichung, an der die Schriftsprache maßgeblichen Anteil hatte, verstärkte sich im 18. Jahrhundert, was zur Stigmatisierung des Apostrophs führte.

Hinzu kommt ab Mitte des 17. Jahrhunderts ein entscheidender Funktionszuwachs des Apostrophs: Er steht häufig als so genannter Genitivapostroph bei Personennamen (Amalia's), markiert also die Grenze zwischen der Grundform (Nom.Sg.) des Namens und dem Flexiv. Dabei zeigt sich folgende belebtheitsgesteuerte Progression: Personennamen $>>$ Ortsnamen $>>$ andere Namen und Appellative (ebd., S. 187). Um die Wende 18./19. Jahrhundert weitet er sich auch auf das Plural-s aus (die Gore's, zwei Sopha's): „Keinesfalls handelt es sich bei diesen Gebräuchen also um Erscheinungen, die zum ersten Mal in der Gegenwartssprache zu beobachten sind" (ebd., S. 188).

Hier mutiert der Apostroph nun zu einem morphographischen (oder logographischen) Zeichen; er wird auch Grenz- oder Stammformapostroph genannt, da er morphologische Grenzen markiert bzw. die Stammform 
auszeichnet und damit schützt. In beiden morphographischen Verwendungen wird er mehrheitlich von den zeitgenössischen Grammatikern (z.B. Grimm, Raumer, Duden) abgelehnt, wiewohl es durchaus einige gibt, die seinen leserseitigen Nutzen erkennen. So schreibt Sanders (1873, S. 30 f.):

Ganz besonders sind hier aber die Eigennamen zu beachten, bei denen es oft von sehr wesentlicher Bedeutung sein kann, sie von den angehängten Flexionstheilen klar und bestimmt geschieden hervortreten zu lassen. Zu diesem Zweck wird dem in der Deklination auftretenden s (gewöhnlich zur Bezeichnung des Genitivs, seltner des Plurals) und dem in der Adjektivbildung auftretenden sch ein Apostroph vorgesetzt, vor welchem der unveränderte Eigenname steht. [...] Orthographisch richtig zu schreiben hat man also nach dem Vorstehenden z.B.: Ein Gedicht Goethe's; Ein Goethe'sches Gedicht [...].

Tatsächlich sind Apostrophe in Texten des 18. und 19. Jahrhunderts überaus häufig. Ewald (2006, S. 149) listet Beispiele von namhaften Autoren auf wie von Humboldt (1797), J. Paul (1800), Schiller (1813), eine Zeitung von 1800. Eindeutig dominieren hier die Eigennamen, besonders solche fremder Herkunft und fremder Struktur (z.B. mit auslautenden Vollvokalen): Galvani's, Creve's, Valli's, Presciani's, Mangili's, Gallini's, Green's, Bilsborrow's, Augusti's, Guerike's, Bell's, Castiglioni's, Gicard's, Coligny's, Katharina's, Beauchamp's, Bobigny's etc., aber auch, wenngleich in der Minderzahl, Lothar's, Heinrich's, Karl's. Ausgangspunkt und Zentrum der morphographischen Apostrophe sind damit eindeutig die Eigennamen, genauer die auf belebte Objekte referierenden Anthroponyme, besonders dann, wenn sie nichtnative Strukturen enthalten.

Nicht zu vergessen ist der so genannte Derivationsapostroph (Ewald 2006), der innerhalb deonymischer Wortbildungen vorkommt, und zwar in Adjektiven auf <'sch>: <Wackernagel'sches Gesetz, Einstein'sche Relativitätstheorie, Grimm'sche Märchen>. Trotz ihres zweifelsfreien Adjektivstatus dürfen diese Derivate groß geschrieben werden; in diesem Fall kann vor das Suffix -sch ein Apostroph gesetzt werden. Diese adjektivische Großschreibung dürfte letztes Relikt historischer Adjektivgroßschreibungen sein, deren Hochzeit um 1700 bestand und deren Kern schon immer deonymische Adjektive bildeten wie < Römisch> (siehe Wegera 1996; Bergmann/Nerius 1998; S. 873-891; Ewald/Nerius 1999). Seit 1996 ist auch die Kleinschreibung deonymischer Adjektive möglich, dann jedoch ohne Apostroph: $<$ grimmsche Märchen>. Das Adjektivsuffix -sch geht aus silbischem -isch hervor (wie es bei kindisch weiterhin besteht). Interessanterweise sind es (wieder) die Personennamen, die hier die Vorreiter sind: Insbesondere dann, wenn eine Zugehörigkeit zum Namenträger bezeichnet wird, steht das kurze Suffix: < Grimm'sche Märchen, Bach'sche Kantaten> (dagegen <lutherisches Bekenntnis $>$ ). Adjektive mit Ortsnamen als Kern, die ebenfalls ein Zugehörigkeitsverhältnis ausdrücken, werden jedoch in aller Regel mit langem -isch abgeleitet, womit eine Apostrophsetzung entfällt: < brandenbur- 
gisch, berlinisch $>.{ }^{2}$ Doch kam es historisch zu (unregelmäßigen) Apostrophsetzungen auch nach Toponymen, allerdings seltener als nach Anthroponymen. Hier zeigt sich der Einfluss der Belebtheitshierarchie beim Sprachwandel (siehe Nübling et al. 2010).

\subsection{Reanalysen}

Beim Funktionswandel vom phono- zum morphographischen Apostroph muss ein Ambiguitätsstadium bestanden haben, in dem die (zunächst leserseitige) Reanalyse vom Auslassungs- zum Grenzzeichen stattgefunden hat. Hier lassen sich sogar mehrere Reanalysen rekonstruieren.

Beginnen wir mit dem letztgenannten Fall des Derivationsapostrophs: Im Fall deanthroponymischer Adjektive, Typ Bach'sche Kantaten, hat eine - offensichtlich morphologisch motivierte, da nicht lautgesetzlich erfolgte Vokalelision von -isch- > -sch- stattgefunden, die, wie jeder Sprachwandel, ein längeres Übergangsstadium in Form von Varianz voraussetzt. Genau hier stand der Apostroph erst (phonographisch) als Auslassungszeichen, nach dem $i$-Ausfall jedoch (morphographisch) als Grenzzeichen:

Mit der Durchsetzung der reduzierten Suffixform dürfte der Derivationsapostroph sukzessive seine Funktion verändert und sich von einem Auslassungssignal zu einem Grenzsignal entwickelt haben. In einem Beleg wie Weygand'sche Buchhandlung (1779) ließe sich der Apostroph folglich zum einen als Auslassungs-, zum anderen als Grenzmarkierung deuten. Mit dieser Entwicklung [...] könnte sich der Derivationsapostroph als wichtiger Wegbahner des Grenzapostrophs erweisen. (Ewald 2006, S. 152)

Im 19. Jahrhundert finden nach Ewald (2006) die ersten Bezugnahmen auf diesen Grenzapostroph statt, z.B. bei Heyse (1849, S. 16, zit. nach Ewald 2006, S. 154), wenn er schreibt: „das Schulze'sche Haus; hingegen: das Schulz'sche Haus od. Schulz'ische Haus, wenn der Mann nicht Schulze, sondern Schulz heißt [...]“. Selbst vor dem vollen Suffix empfiehlt er die Apostrophschreibung, was den morphographischen Status umso stärker unterstreicht (allerdings war die Apostrophsetzung vor -isch, wie Ewald 2006, S. 155-157 nachweist, auch im 18./19. Jahrhundert nicht üblich). In seiner Begründung rekurriert Heyse nicht etwa auf die morphologische Grenze, sondern auf die erkennbar bleiben sollende Grundform des Namens. Insofern ist der Begriff des logographischen Apostrophs durchaus gerechtfertigt (Klein 2002). Zentral ist die wortkörperschonende Funktion des Apostrophs. Sie spielt nicht nur in der graphematischen, sondern, wie wir in Abschnitt 3 sehen werden, auch in der morphologischen Entwicklung

Natürlich auch hier mit Ausnahmen: Hannover'sche/Hannoversche Politik, Hannover'sche Maschinenbau-Actien-Gesellschaft, Hannoversche Allgemeine, hannoverscher Schweißhund. Möglicherweise spielt hierbei der Namenauslaut eine Rolle. 
der Namen eine entscheidende Rolle. Wie Ewald (2006, S. 157) herausstellt, setzen sich diese Apostrophe unabhängig von zeitgenössischen orthographischen Regeln und Empfehlungen durch: „Der Apostroph erweist sich somit im 18./19. Jh. als eher regelungsresistentes Orthogramm“ (ebd., S. 158). Umso mehr spricht dies für seine Funktionalität.

Auch beim Genitivapostroph ist die reanalysefördernde Brücke zwischen phono- und morphographischer Funktion rekonstruierbar: Im Laufe der Sprachgeschichte wurde das ursprünglich ausschließlich lange (silbische) Genitiv-es unter bestimmten Bedingungen zu unsilbischem -s synkopiert (ahd./mhd. Hartmuotes > nhd. Hartmuts); die genaue Entwicklung, ihre prosodisch-phonologische Bedingtheit sowie die Ratio dahinter findet sich bei Szczepaniak (2010). Auch die Eigennamen waren von dieser Synkope betroffen, wenngleich hier eine (nur morphologisch zu begründende) Beschleunigung stattgefunden haben muss, denn heute gilt für Eigennamen (wie auch für Fremd- und Kurzwörter) prinzipiell, dass sie nur das kurze Genitiv-s nehmen, unabhängig von den sonst geltenden Faktoren. Lange Endungen oder mögliche Varianz zwischen beiden Allomorphen ist hier nicht mehr möglich, vgl. appellativisch des Kind(e)s, aber onymisch Süskinds (*Süskindes) Werk; ebenso: die Blätter des Busch(e)s, aber Wilhelm Buschs (*Busches) Gedichte. Klein (2002, S. 188) schreibt hierzu:

Was den Genitiv-Apostroph betrifft, so scheint auch er anfangs eine mehr oder weniger deutliche phonographische Fundierung zu besitzen: Synkopierte einsilbige Formen wie $<$ Gott's Wahrheit $>$ wurden offensichtlich von den Dichtern genutzt, weil die zweisilbigen Formen (<Gottes $>$ ) nicht ins Versmaß passten. Möglicherweise ausgehend von solchen phonographischen Schreibungen wurden dann aber schon in der Mitte des 17. Jahrhunderts Eigennamen manchmal mit dem Genitiv-Apostroph geschrieben.

Wann genau die Eigennamen die radikale Synkope von genitivischem -es $>-s$ vollzogen haben, ist nicht genau bekannt (Namen werden aus vielen Untersuchungen ausgeklammert). Ausgehend vom $s$-Genitiv hat sich der Apostroph sporadisch, ab Ende des 18. Jahrhunderts, auf den s-Plural übertragen, hier jedoch von Anfang an als morphographisches Zeichen. Klein (ebd., S. 189) macht eher beiläufig eine wichtige Beobachtung: „Er ging aus von Familiennamen und verbreitete sich dann insbesondere bei Fremdwörtern." Nach Nübling/Schmuck (2010) geht der $s$-Plural, der als solcher tatsächlich bei den Familiennamen startet, auf die Reanalyse des Genitiv-s zum Pluralmarker zurück. Diese immer wieder geäußerte These wird hier sowohl für das Deutsche als auch das Niederländische erhärtet. An der Stigmatisierung dieser $s$-Endung durch zeitgenössische Grammatiker kann man ihr ungefähres Alter ablesen. So schreibt Blatz (1900, 336 f.):

Der Plural bei Personeneigennamen wird mittelst der Endungen -e, -ne, -en (n), -nen gebildet, z.B. Die beiden Stolberge [...]. - Nicht gut: die beiden Schlegels, Heindorfs. Anders: Benders verreisen heute (elliptischer Genet. Sing.). 
Anders ist im letzten Beispiel gar nichts; es handelt sich bei Benders ebenso um einen Plural wie bei Schlegels und Heindorfs, erkennbar am Verbalplural verreisen. Was für Blatz offensichtlich noch durchscheint, ist die alte (als elliptisch empfundene) Ursprungskonstruktion (des) Benders Leute/Familie, aus der heraus die Reanalyse vom Genitiv Singular zum Nominativ Plural stattgefunden hat. In heutigen Dialekten finden sich noch erstarrte Reliktkonstruktionen wie z.B. alemannisch $s$ Benders sin do, die (wörtl.: ,des') Benders sind da', ich bin bi s Benders, ich bin bei (des) Benders', ich gang zu s Benders, ich gehe zu (des) Benders' etc. Dem Familiennamen vorangestelltes $s$ tradiert noch den alten Gen.Sg.-Artikel, doch fordert und bezeichnet die NP längst den Plural (zu dieser Reanalyse siehe eingehend Nübling/Schmuck 2010; Schmuck 2011). So nimmt es nicht wunder, dass die Familiennamen nicht nur Entstehungsort des $s$-Plurals, sondern auch des $s$-Pluralapostrophs sind. Eigennamen bilden oft das Zentrum sprachlicher Innovationen und werden in dieser Funktion sowohl von der Sprachgeschichts- als auch der Grammatikschreibung unterschätzt (mehr hierzu in Abschnitt 3).

Die weiteste Entwicklung hat der Apostroph im Falle der auf [s] auslautenden Namen wie Hans, Ines, Marx, Fuchs, Schmitz im Genitiv zurückgelegt. Hier lässt sich das Genitiv-s nicht artikulieren, da es keine auslautenden Geminaten gibt (die ältere Endung -ens, vgl. Hansens Mutter, ist heute kaum noch gebräuchlich). Ersatzweise steht hier der Apostroph. Da er keinerlei phonographische Funktion versieht, auch kein Morphem vom Stamm abhebt, ist er hier selbst zu einem Suffixersatz, zu einem rein graphischen Morphem aufgestiegen; in Hans' Geburtstag, Ines' Abitur hat er morphologischen und damit grammatischen Status erlangt. Das, was das Französische kennzeichnet (stumme Grapheme mit grammatischen Funktionen), ist damit auch im Deutschen an dieser Stelle vorhanden. Man kann den Apostroph auch ausschließlich als rechtes Grenzzeichen für die onymische Grundform (den Nom.Sg.) sehen und ihm damit logographische Funktion zuweisen. Er signalisiert dann die Integrität des Wortkörpers wie bei <Ines', Hans'>, d.h. er zeigt an, dass das auslautende $-s$ nicht als Genitivendung von $*<$ Ine, $*$ Han $>$ fehlzudeuten ist. In Fällen wie <Andrea's $>$ vs. <Andreas'> (auch $<$ Carlo's $>/<$ Carlos' $>$ ) werden phonologische Homonyme graphematisch disambiguiert. Hier jedoch eine tiefenstrukturell anzusetzende phonologische Geminate (*-[s:] im Auslaut verbietet sich im Dt.) graphematisch angezeigt zu sehen, ist wenig plausibel, da dieses Prinzip nirgendwo sonst im Deutschen Verwendung findet (vgl. sie * rät'/*brät'; siehe Ewald 2006, S. 142).

Eine dritte, jüngere Brücke zwischen phono- und morphographischem Apostroph liefern die Präposition-Artikel-Verschmelzungen, solange diese noch ohne Bedeutungsveränderung mit ihrer Vollform austauschbar sind. Dies gilt z.B. für das Autor steht vor'm (= vor dem) Haus, sie kommt mit'm (= mit dem) Fahrrad, doch nicht für wir fahren ins (*in das) Engadin, wir sind beim (*bei dem) Papst (Nübling 2006). Bei der ersten (austauschbaren) Gruppe setzt 
man den Apostroph, bei der zweiten nicht; hier gelten die Verschmelzungen (zurecht) als „gebräuchlich“, denn sie sind fest grammatikalisiert. Der Apostroph in vor'm und mit'm kann sowohl phonographisch als Auslassungszeichen für die Sequenz de-interpretiert werden als auch morphographisch als morphologische Grenze zwischen Präposition und Artikel.

Zusammenfassend lässt sich die Evolution des Apostrophs mit Abbildung 2 skizzieren. Insgesamt fügt sich seine Entwicklung voll und ganz den immer leserfreundlicheren, sich sukzessive vertiefenden Verschriftungsprinzipien des Deutschen. Dabei behält er auch seine alten Funktionen bei, sogar die als echtes Kürzelzeichen bei Toponymen (Typ D'dorf) ${ }^{3}-$ ebenso wie generell das Deutsche trotz seiner Überformung durch zahlreiche morphosemantische Schreibprinzipien nach wie vor eine stark phonographisch ausgerichtete Schreibung praktiziert.

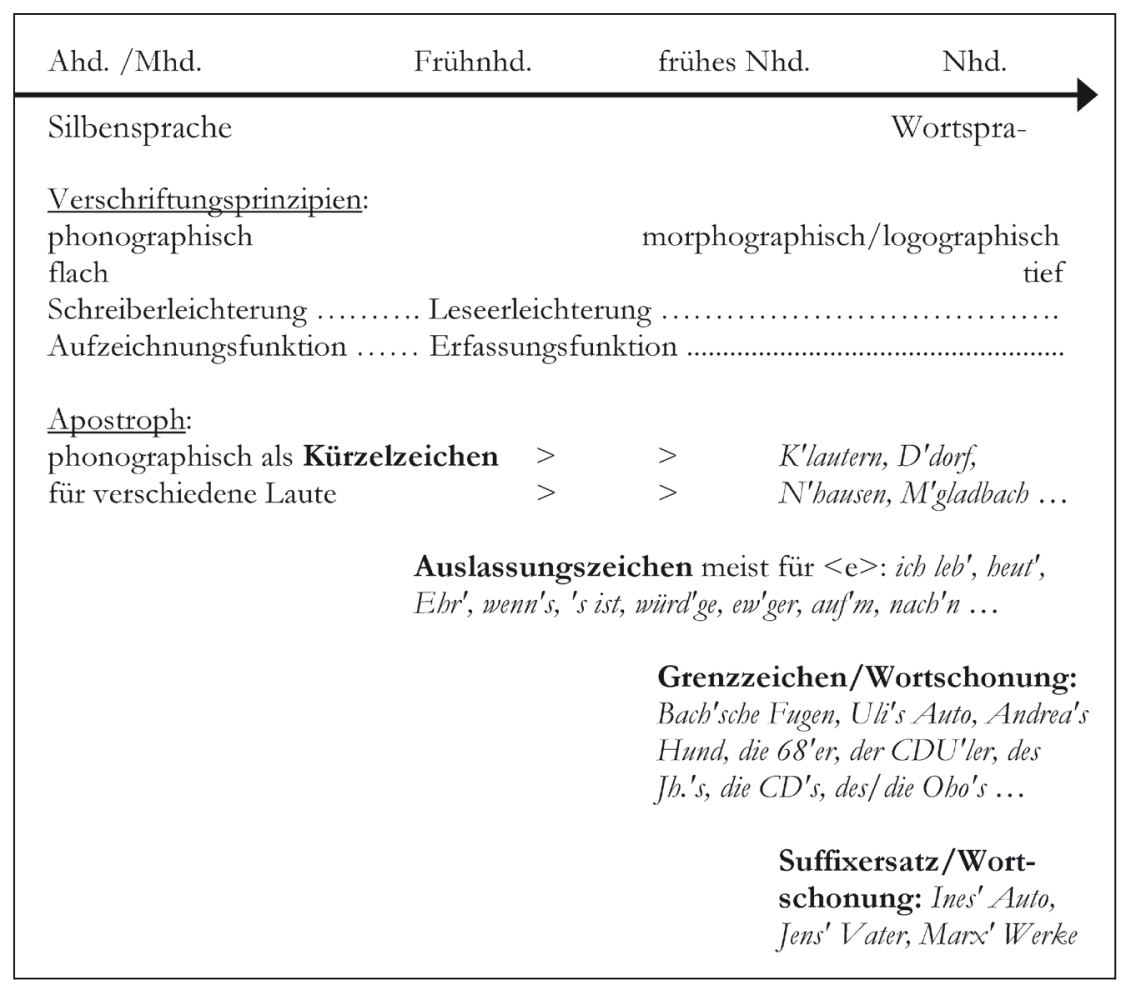

Abb. 2: Die Evolution des Apostrophs zum morphographischen Zeichen

Selbst die ursprüngliche Funktion der Kürzel, den Satzspiegel einhalten zu können, ist hier noch gegeben: Abkürzungen wie D'dorf und K'lautern finden sich v.a. in Tabellen oder auf Straßenschildern mit wenig Platz. 
Jenseits der Norm kommt es heute zu weiteren morphographischen Apostrophverwendungen, z.B. bei Dialektverschriftungen, wo der Apostroph beim Definitartikel $s<$ das nicht dort, wo die Auslassung erfolgt, steht ('s Eck), sondern oft dort, wo die morphologische Grenze zwischen Proklitikon und Basis verläuft: $s^{\prime} E c k$. Sehr oft grenzt der Apostroph Akronyme (Buchstabierkurzwörter) vom flexivischen Rest ab (die CD's, des $P K W ' s$ ), ebenso (graphische) Abkürzungen (C.'s Hund, A.'s Haftzeit) oder gar Logogramme wie Zahlen (die 68'er). In allen Fällen wird die Stammform, d.h. das Lexem bzw. der Namenkörper, geschützt.

\subsection{Stigmatisierung und heutige Regelung}

Es dürfte wohl kaum einen Sprachwandel geben, der nicht durch seine Zeitgenossen (die ihn selbst vorantreiben) gegeißelt und als Sprachverfall abgewertet würde. Das ist heute nicht anders als früher. Nach wenigen Generationen sind jedoch die alten guten Zeiten bzw. Formen vergessen: Niemand möchte heute zum Ahd. zurückkehren; es trauert auch niemand mehr einst starken Verben wie pflegen, bellen oder salzen nach, doch durchaus backen, flecbten oder melken, da letztere gerade dabei sind, diesen Wechsel von stark zu schwach zu vollziehen. Im Fall des Apostrophs hat seine Stigmatisierung jedoch eine ungewöhnlich lange Tradition, sie findet sich schon im frühen 18. Jahrhundert. Klein (2002) und Ewald (2006) zeichnen die Geschichte der Missbilligung des Apostrophs nach. Besonders der (neue) morphographische Apostroph wird am schärfsten kritisiert (die wenigen Befürworter, die seine Funktion erkannt haben, sind Heyse 1849 und Sanders 1873, siehe Abschnitt 2.1). Stellvertretend für viele sei Wustmann (1891, S. 54 f.) zitiert, der sogar gegen jegliche Apostrophsetzung polemisiert:

Goethes oder Goethe's? Großes Vergnügen macht es vielen Leuten, den Genitiv von Personennamen mit einem Apostroph zu versehen: Friedrich's, Müller's. Selbst große Gelehrte sind in den Apostroph so verliebt, daß es ihnen ganz undenkbar erscheint, Goethes ohne das hübsche Häkchen oben zu schreiben. Nun ist der Apostroph überhaupt eine große Kinderei. Alle unsre Schriftzeichen bedeuten doch Laute, die gesprochen werden [...] Einzig und allein der Apostroph bedeutet gar nichts; ja er soll geradezu einen Laut bedeuten, der - nicht da ist [...]. Ist das nicht schon kindisch?

Besonders vehement geht er gegen den Apostroph als Suffixersatz bei Namen auf [s] vor, wo er sowohl $*<$ Leibniz's Plan $>$ als auch $*<$ Leibniz' Plan $>$ ablehnt („,gar zu einfältig“). Hier möchte er gerne die lange ens-Endung reaktivieren: Leibnizens Plan. Doch ist er wiederum gegen Brabmsens Sonate und Sophoklessens Antigone (,klingt nicht schön“); hier rät er gar, den Namen zu vermeiden: des Dichters Werk oder sein Werk.

Auch auf der 1. Orthographischen Konferenz von 1876 stellte sich Konrad Duden vehement gegen den sich „eingeschlichen“ habenden Apostroph 
und verhinderte, dass er in seiner morphographischen Funktion als Grenzsignal ins Regelwerk einging. Einzig als Auslassungszeichen (beil'ge Nacht), worunter hier auch das eigentlich zu realisierende Genitiv-s bei Namen auf [s] fällt (Voß’ Luise), ist er seit 1901 erlaubt. In seinen morphographischen Funktionen vor den Suffixen -s und -sch-ist er untersagt (also: Andreas Hund, Schillersche Dramen). Erst in der Reform von 1996, die den Apostroph in

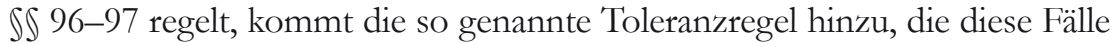
zumindest nicht als regelwidrig ausweist (was sie vorher eindeutig waren) und die sich wie folgt liest (als Ergänzung zu \ 97):

E: Von dem Apostroph als Auslassungszeichen zu unterscheiden ist der gelegentliche Gebrauch dieses Zeichens zur Verdeutlichung der Grundform eines Personennamens vor der Genitivendung -s oder vor dem Adjektivsuffix -sch: Carlo's Taverne, Einstein'sche Relativitätstheorie.

Dies ist keine Aufforderung oder gar Verpflichtung, vor dem Genitiv-s bzw. dem Suffix -sch- einen Apostroph zu setzen, sondern lediglich eine Erlaubnis, dies zu tun. Auch lässt das einzige Beispiel Carlo's offen, ob dies nur bei fremden Namen bzw. solchen, deren Grundform auch ein festes, auslautendes $-s$ enthalten könnte, erlaubt ist (Carlos ist auch ein spanischer Vorname), ob also eine Disambiguierungsfunktion enthalten sein muss (Ewald 2006, S. 143). Dies alles bleibt im Dunkeln. Die Schonung der Grundform bildet die einzige leitende Absicht. Der Apostroph vor Plural-s und generell nach Appellativen wird jedoch nirgends erwähnt, also bleibt er hier normwidrig.

$\int 62$ regelt die deonymischen Adjektivableitungen und schreibt bei ihrer Großschreibung Apostrophsetzung vor, was eine Neuerung ist. Ebenfalls neu ist die Möglichkeit ihrer (apostrophfreien) Kleinschreibung:

Kleingeschrieben werden adjektivische Ableitungen von Eigennamen auf -(i)sch, außer wenn die Grundform eines Personennamens durch einen Apostroph verdeutlicht wird, ferner alle adjektivischen Ableitungen mit anderen Suffixen. Beispiele: die darwinsche/ die Darwin'sche Evolutionstheorie, das wackernagelsche/Wackernagel'sche Gesetz [...].

Ansonsten (siehe \96) ist der Apostroph in diversen Auslassungsfunktionen weiterhin zugelassen (auch in seiner genuinsten und ältesten Funktion als Kürzel, wo er für unterschiedliche zu artikulierende Laute steht, Typ D'dorf). Allerdings findet sich der Zusatz, dass Wörter ohne ihn ,schwer lesbar oder missverständlich" sein müssen, z.B. in wen'gen Augenblicken, das Wasser rauscht'. Seine Verwendung als Auslassungszeichen wurde eingeschränkt, etwa bei häufigen Apokopen (ich leb, ich geh), Synkopen (andre, wechsle), Imperativen (komm, lass) und gebräuchlichen Präposition-ArtikelEnklisen (ins, überm, übern). \97 erlaubt ihn nur bei ungewöhnlichen Enklisen wie mit'm, das war'n (= war ein) Erfolg, wo „Wörter gesprochener Sprache mit Auslassungen bei schriftlicher Wiedergabe undurchsichtig sind“" ( $\left.\int 97\right)$. Wie sich jedoch Undurchsichtigkeit und schwere Lesbarkeit definieren, dies 
alles bleibt unklar. Die Formulierungen sind eher implizit als explizit und enthalten mal Muss-, mal Kann-Bestimmungen. Man merkt auf Schritt und Tritt, wie heikel dieses Thema ist (siehe auch Klein 2002, S. 190-193).

Wie bzw. warum kam es zu dieser langen Geschichte der Stigmatisierung bis hin zu dafür eigens eingerichteten Websites? Diese Frage beantwortet eingehend Klein (2002): Letztlich geht die Geringschätzung des Apostrophs auf sein frühes phonographisches Stadium als Auslassungszeichen im 17./18. Jahrhundert zurück, da er - zu Zeiten, als der Wunsch nach einer überregional gültigen Standardsprache aufkam - dialektale bzw. als substandardsprachlich empfundene, reduzierte Formen der gesprochenen Sprache markierte. Wörter mit Auslassungsapostroph setzen immer ein „vollständiges" Vergleichswort voraus, das eigentlich gemeint ist und der (statushohen) Schriftsprache angehört; damit weisen sie sich selbst als defizitär aus. Gallmann (1985, S. 258) sieht hierin „eine der eigenartigsten Funktionen eines Graphems in unserm Schriftsystem" und bezeichnet den Auslassungsapostroph als „Normverstoßsignal“. Klein (2002, S. 176) charakterisiert den Apostroph als relationales Syngraphem, da er auf zwei Formen rekurriert, die reduzierte und die volle. Aus dieser Differenz, der Spannung zwischen Standard und Standardferne, dürfte die prinzipielle Negativbewertung des Apostrophs herrühren. Die Schreiberin selbst drückt damit ihr Wissen um die „richtige“ Form aus, was den Apostroph nach Klein auch zu einem stilistischen Syngraphem macht. Fällt eine bisherige Apostrophsetzung weg (wie bei geläufigen Enklisen, beispielsweise durchs, vorm, oder Phraseologismen, zum Beispiel mit Müh und Not), begreift man die betreffende Form quasi als standardsprachlich geadelt: Sie ist aufgestiegen.

\subsection{Die Apostrophsetzung in gegenwartssprachlichen Korpora}

Außer impressionistischen Äußerungen, oft basierend auf Zufallsfunden und kleineren Sammlungen, gab es bis 2010 keine korpusbasierten Untersuchungen zur Apostrophsetzung in heutigen Texten. Mit Bankhardt (2010) und Scherer (2010) sind fast gleichzeitig zwei Arbeiten erschienen, jüngst auch Scherer (2013). Diese Ergebnisse sollen nun resümiert werden, wobei besonderes Gewicht auf die jüngeren, von Vielen kritisierten morphographischen Apostrophe gelegt wird.

Bankhardt (2010) hat Dezemberausgaben des „Mannheimer Morgens“ von 2004 synchron untersucht. Erfasst wurden sämtliche Apostrophe, was 914 Treffer ergab. Davon entfallen 695 auf Elisions- (76\%) und 190 (20\%) auf morphographische Apostrophe (der Rest sind Abkürzungen wie $<$ N'hausen $>$ ).

Scherer (2010, 2013) hat drei große Korpora untersucht: 1. das DWDS des 20. Jahrhunderts, womit sie die Diachronie von ca. 100 Jahren erfasst, 2. die Berliner Zeitung (BZ) von 1994/95 sowie 3. die Potsdamer Neuesten 
Nachrichten (PNN) von 2003-2005. Extrahiert hat sie die Sequenz <'s>, d.h. sie erfasst ausschließlich Apostrophe vor $s$ am Wortende. Nicht erfasst werden also die Adjektive vom Typ Einstein'sche Theorie und die Eigennamen auf $-s$ vom Typ Ines' Hund. Sie gelangt zu insgesamt 23.810 Belegen, wovon insgesamt $20.567(=86 \%)$ auf Elisions- (inkl. Verschmelzungen wie $<$ auf's $>$ ) und $3.243(=14 \%)$ auf morphographische Apostrophe entfallen. Was die Entwicklung der letzten 100 Jahre betrifft, so sinkt der Anteil der Elisionsapostrophe in Zeitungstexten (bei allem textsortenbedingtem Auf und Ab) insgesamt um 10-20 Prozentpunkte ab (DWDS 1900-1909: 85\%, 1910-1919 sogar 95\%; BZ 1994-95: 77\%, PNN 2003-05: 74,3\%). Dies geht zugunsten der morphographischen Apostrophe: Im DWDS 1900-1919 befinden sie sich noch im einstelligen Prozentbereich, heute (BZ, PNN) bei ca. 21\% (siehe Scherer 2010, S. 6-8).

Uns interessieren im Folgenden nur die morphographischen Apostrophe, d.h. deren genaue Funktionen und Basen.

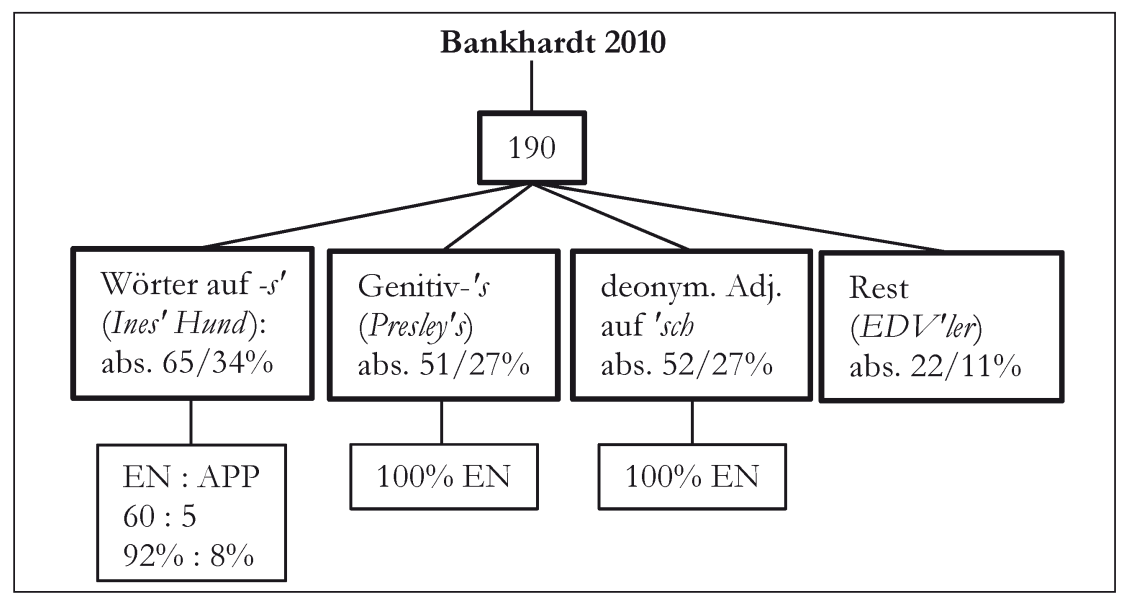

Abb. 3: Bankhardt (2010): Die vier Funktionsbereiche des morphographischen Apostrophs (Suche: alle $<>>$; Tokens)

Abbildung 3 enthält Bankhardts Befunde, die einen überaus klaren Schluss nahelegen: Es sind zuvörderst Eigennamen, deren Flexive durch einen Apostroph vom Namenkörper abgegrenzt werden. In seiner Funktion als Suffixersatz (Typ Ines' Hund) kommt der Apostroph zu 34\% vor, dabei zu über 90\% an Eigennamen, genauer: Personennamen. Vor einem Genitiv-s steht er zu 27\%, hier ausschließlich nach Eigennamen, genauer: fast nur Personennamen. Exakt das Gleiche gilt für die deonymischen Adjektive auf 'sch. Die verbleibenden 22 Apostrophe (11\%) enthüllen auch eine klare Funktion: Ihr größter Basenanteil sind Akronyme, die personenbezeichnende Derivate auf -ler bilden (Typ DRK'ler). Hier leistet der Apostroph sogar eine 
doppelte Abgrenzungsfunktion: einerseits die Bezeichnung der morphologischen Grenze zwischen Stamm und Suffix, andererseits die zwischen Syllabo- und Phonographie, wobei die Syllabogramme zusätzlich durch Majuskeln markiert werden (Gallmann 1985, S. 101). In Anlehnung an Gallmann (1985) nennt Scherer (2010) dies das graphematisch-morphologische Prinzip. Würde das gesamte Wort (z.B. in einer Überschrift) großgeschrieben (DRK'LER), wäre der Apostroph sogar das einzige Grenzsignal für die beiden Schriftsysteme. Übrigens hat auch ein Teil dieser Akronyme Eigennamenstatus (z.B. DRK). Selbst die sechs hinzukommenden Apostrophe vor dem Plural-s folgen ausnahmslos Akronymen bzw. Kurzwörtern (LP's, GI's, KiK's). Von irrlichternden „Deppenapostrophen“ kann nicht die Rede sein, auch wenn die Pluralapostrophe normwidrig sind.

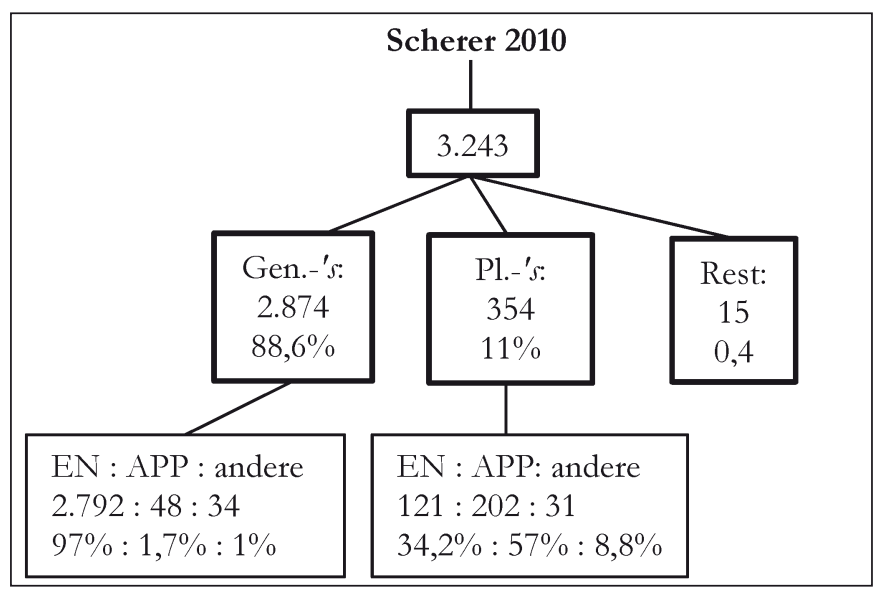

Abb. 4: Scherer (2010): Die beiden Funktionsbereiche des morphographischen Apostrophs (Suche: alle $<$ 's $>$ am Wortende; Tokens)

Auch Scherer (2010, 2013) erbringt den Nachweis, dass regelwidrige Setzungen in ihrer Frequenz weit überschätzt werden und, indem sie auch eine diachrone Perspektive enthält, dass es sie schon immer gab. Abbildung 4 enthält ihre Ergebnisse, die auf weit größeren Zahlen basieren als die von Bankhardt.

Knapp 89\% der Apostrophe vor finalem 's grenzen ein Genitiv-s ab. Auch hier reicht der Anteil der onymischen Basen an die 100\%-Marke heran (es sind knapp 98\%). Abbildung 5 zeigt eindrucksvoll, dass allein mit 94\% die Personennamen das Gros ausmachen. Darunter fallen primär Familiennamen, dann Rufnamen und schließlich weitere Personennamen (wie Mao, Buddha, Nessi). ${ }^{4}$

$4 \quad$ An Carmen Scherer geht mein großer Dank dafür, dass sie mir das gesamte Korpus zur Verfügung gestellt hat, ebenso die Graphik in Abbildung 5. 


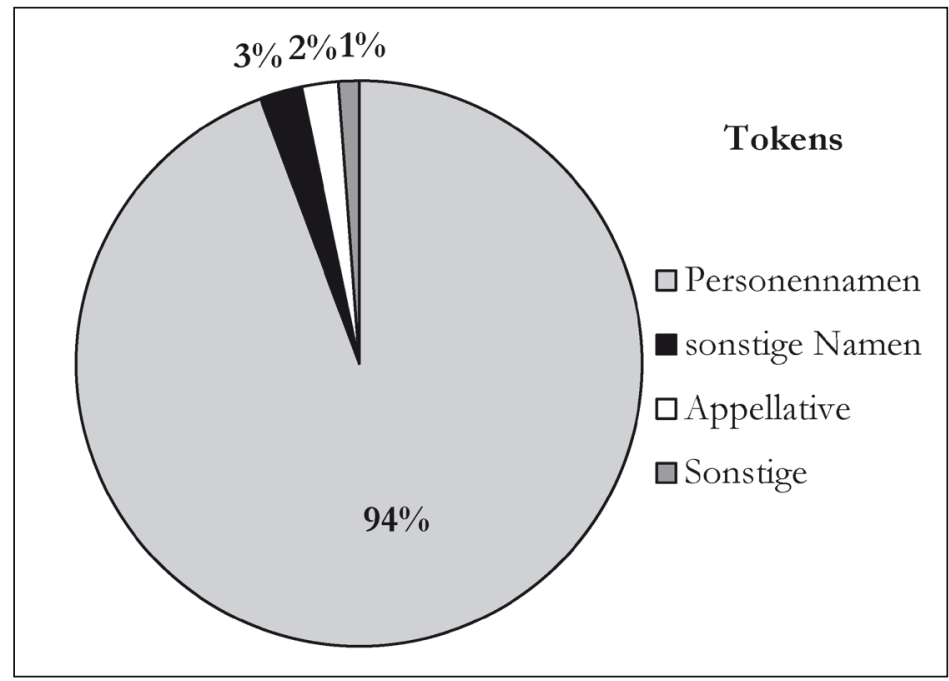

Abb. 5: Apostroph vor Genitiv-s im Scherer-Korpus: Basen

Die höchste Wahrscheinlichkeit der Apostrophsetzung besteht dann, wenn zwei (oder mehr) begünstigende Faktoren zusammentreffen, z.B. onymischer Status + Abkürzung (mit und ohne Abkürzungspunkt), Typ Joseph K.'s, S.B.'s., F.'s/F's. Hier ist die Apostrophsetzung normwidrig. Diese Gruppe ist im Korpus überraschend groß, wohl deshalb, weil sie eher Apostrophsetzung erzwingt als ungekürzte Eigennamen. Da die s-Genitive ohne Apostrophsetzung nicht erfasst wurden, lässt sich nicht sagen, um wieviel häufiger gekürzte Namen im Genitiv apostrophiert werden als ungekürzte. Doch legt der überaus hohe Anteil abgekürzter Namen mit Apostroph im Scherer-Korpus die Vermutung nahe, dass dies Usus ist (Näheres siehe Scherer 2010, S. 9-14).

Mit 3\% relativ gering ist die Gruppe der ,,sonstigen Namen“, worunter v.a. Toponyme fallen, die, wie das Korpus ausweist, auffällig viele Fremdmerkmale enthalten: Benomatapa, Moskwa, Inyanga, Pozuzo etc. Hier greift der dritte apostrophfördernde Faktor, nicht-native Strukturen, also Fremdwortcharakter. Belebtheit ist bei den Personennamen das wichtigste Kriterium, das Apostrophsetzung zur Namenkörperschonung auslöst. Dies schließt keineswegs aus, dass Kurz- oder Fremdwortmerkmale ihrerseits die Apostrophsetzung verstärken; ein Blick ins Korpus bestätigt dies. Je mehr sich die Basis jedoch von diesem Prototyp entfernt, desto eher müssen andere begünstigende Faktoren hinzutreten. Besonders deutlich zeigen dies die 1,7\% der Appellative (= 60 Tokens), die kategorial am weitesten von den Personennamen entfernt sind, aber umso mehr fremde bzw. kurzwortige Strukturen enthalten: Bureau, Bialy, Joker, Genie, Raver, Majestät, EEG, KB, LP, 
LKW, Pkw etc. Damit erweist sich, dass morphographische Apostrophe primär wortkörperschonende Funktionen ausüben (was Scherer 2013 nach Gallmann 1985 als pragmatisch-morphologisches Prinzip bezeichnet). Der so genannte Rest (in Abb. 4), der auf die Genitivapostrophe entfällt, umfasst 34 Tokens, die sich auf genau drei Types verteilen, ausnahmslos Abkürzungen: <Jh's, A's, B's>.

Die zweite Domäne der Apostrophsetzung vor wortfinalem -s betrifft das Plural-s (Scherer 2010, S. 14-18). Dies macht 11\% der morphographischen Apostrophe aus (siehe Abb. 4). Obwohl Eigennamen, da monoreferent, selten in den Plural treten (nur dann, wenn es mehrere Referenten mit gleichem Namen gibt), machen sie in dieser Gruppe immer 34\% aus gegenüber 57\% Appellativen und 8,8\% anderen Wörtern. Die Namen verteilen sich fast ausschließlich auf Familiennamen (Typ die Buchbolzen's), wobei sich hierunter mehr abgekürzte als volle Namen befinden (häufigster Typ: die M.'s/die M's). Genauer: zwei Drittel sind abgekürzt, nur ein Drittel nicht. Selbiges betrifft die Appellative: Wenn sie nicht aus Fremdwörtern bestehen (z.B. die Dandie's, Kotelett's), dann handelt es sich auch hier (mehrheitlich) um Abkürzungen (die LP's, $L K W$ 's), v.a. wenn sie fremdsprachlich sind (die DJ's, GI's). Man sieht: Die Zutrittsbedingungen zu einem apostrophierten Plural-s sind deutlich höher als beim Genitiv, meist sind die Wörter mehrfach motiviert. Unter ,,andere“ fallen Konversionen (die Ach's, Hm's, Etc's) sowie einbuchstabige Musiknoten, Variablen (die W's) u.Ä. Kurzum: Unter den 354 Belegen befindet sich kein einziges genuines, natives, ungekürztes Appellativ (solche Appellative nehmen jedoch üblicherweise ohnehin kein Plural-s).

Schließlich ist in Abbildung 4 ein kleiner Rest von 15 Tokens verzeichnet mit nur 0,4\% Anteil an allen morphographischen Apostrophsetzungen. In dieser marginalen Gruppe tummelt sich nun das, was in der Öffentlichkeit als grassierende Deppenapostrophe wahrgenommen wird: Derivationsaffixe wie Fugenelemente (Kalb's Leber, Bahnhof's Gaststätte), -s zur Adverbbildung (dienstag's, besonder's) oder vermeintlich für einen Genitiv gehaltene Namen wie Phil Collin's. Auch wenn dies zweifellos normwidrige Setzungen sind, so zeugen sie doch von einer schreiberseitigen Reanalyse als Genitiv-s, sie folgen also einer Regel(übergeneralisierung). Im Fall einiger Komposita (Kalb's Leber) resyntaktisieren sie sogar diese feste morphologische Verbindung zu attributiven Genitivphrasen. Gerade solchen neueren Apostrophsetzungen geht ausführlich Scherer (2013) nach, u.a. auch mit Internetrecherchen. Sie sieht hierin eine interessante funktionale Weiterentwicklung des morphographischen Apostrophs: Diente er anfänglich ausschließlich der Auszeichnung der rechten Grenze des Wortstamms und damit seiner Schonung (Lafontaine's), so wandelt er sich gegenwärtig dazu, die linke Morphemgrenze zu markieren, d.h. das $<_{\mathrm{s}}>$-Segment gut erkennbar zu halten und 
abzugrenzen von einer in aller Regel wohlgeformten Wortform links davon (niemal's, fall's, namen's, seiten's etc.), die diese (fehlerhafte) Reanalyse auslöst. Auffälligerweise tritt der morphographische Apostroph (so gut wie) nie vor das silbische Genitivallomorph -es (z.B. *<des Bild'es $>$ ) und sehr selten vor $\langle\mathrm{n}>,<$ isch $>$ oder $<$ ler $>$. In jedem Fall sind die Schreibenden auch solcher norm-, wenngleich nicht regelwidriger Apostrophe an der leserseitigen Erleichterung der Wortdekodierung interessiert, sie geben Auskunft über die (aus ihrer Sicht geltende) Wortstruktur. Damit verhält sich die Apostrophsetzung (auch die normwidrige) nicht unsystematisch. Dies zeigt, dass morphologische Verschriftungsprinzipien bis heute produktiv sind und dass sie in einen Zusammenhang zu stellen sind mit vermehrter Bindestrichschreibung bzw. auch Spatiensetzung bei Komposita (<Jod-Salz $>$ bzw. <Jod Salz>) oder der Verwendung so genannter Binnenmajuskeln $(<$ JodSalz $>$ ). Nicht zufällig finden sich auch diese neueren (ebenso negativ bewerteten) Schreibungen vorrangig bei Eigennamen. Eigennamen bilden somit das Zentrum dieser Neuerungen, insbesondere die die Spitze der Belebtheitshierarchie besetzenden Personennamen. Doch haben Eigennamen nicht nur die morphographische Apostrophschreibung begründet und gesteuert, auch andere Sprachwandelerscheinungen haben hier begonnen, z.B. die erwähnte Herausbildung des Plural-s über die Reanalyse des Gen.Sg.-s bei kollektiv verwendeten Familiennamenkonstruktionen, die Entstehung und Ausbreitung von Diminutivsuffixen, die Herausbildung und Durchführung der Monoflexion in der NP sowie der Deflexion am Wortkörper selbst. Sogar das deutsche Alleinstellungsmerkmal der Substantivgroßschreibung hat sich an dieser Belebtheitshierarchie orientiert und begann bei den Eigennamen (Bergmann 1999; Nübling et al. 2010, S. 195-199; Szczepaniak 2011). Wie Schmuck (2009) zeigt, fungieren Personennamen sogar als Quelle von Grammatikalisierungen. Eigennamen haben damit schon vielfach Sprachwandel in Gang gesetzt. Dies steht ihrer Marginalisierung sowohl in der Grammatik- als auch in der Sprachgeschichtsschreibung diametral entgegen. Zu oft werden sie hier ausgeblendet bzw. in Anmerkungen oder Fußnoten gesteckt.

\subsection{Die Apostrophsetzung als Entlehnung aus dem Angelsächsischen?}

Die weit verbreitete Annahme, die (morphographische) Apostrophschreibung entstamme (ähnlich wie das Plural-s) dem Englischen, kann schnell zurückgewiesen werden: Den Beginn des englischen Lehneinflusses setzt man mit 1945 an. Wie gezeigt, ist der umstrittene morphographische Apostroph jedoch schon im 18. Jahrhundert im Deutschen vorhanden, und dies mit einer Frequenz und Geläufigkeit, die oft übersehen wird. Außerdem werden im Zuge dieses Sprachkontakts ab 1945 v.a. Lexeme entlehnt. Die 
Grammatik bleibt hiervon unberührt. Dass es bei diesem Kontakt zur Entlehnung einer graphematischen Regel („Apostroph vor Genitiv-s“) kommt, setzt einen weitaus intensiveren Sprachkontakt voraus. Außerdem deckt sich bei genauerem Hinsehen die deutsche Apostrophsetzung nicht mit der englischen, denn im Englischen steht der Apostroph vor jeglichem Genitiv-s, auch bei Appellativen (vgl. my brother's house, vgl. jedoch nhd. das Haus meines *Bruder's). Native Appellative bilden im Deutschen gerade diejenige Domäne, bei der die Apostrophsetzung am verlässlichsten unterbleibt. Wie deutlich wurde, beschränkt sich der Apostroph primär auf Eigennamen und sekundär auf anderweitig markierte Wortstämme (Fremdwörter, Kurzwörter, Konversionen, nichtphonographische Verschriftung der Basis). Auf den zweiten Verdachtsfall, dass auch das Plural-s dem Englischen entstamme, geht das folgende Kapitel ein.

\section{3. $\quad s$-Plural, $s$-Genitiv und Deflexion als weitere Wortschonungsstrategien}

Abschließend soll gezeigt werden, dass der Apostroph in seiner wortschonenden Funktion graphematischer Reflex jahrhundertelanger morphologischer Entwicklungen des Flexivs selbst ist, das seinerseits der phonologischen Wortschonung dient. Es ist kein Zufall, dass ausgerechnet $-s$ sich sowohl zum onymischen Genitiv- als auch Pluralmorphem entwickelt hat - und dass es heute schwindet.

Auch der s-Plural wird gerne englischem Einfluss zugeschrieben. Sobald man jedoch einen Blick auf die Sprachgeschichte wirft, wird offenkundig, dass das Plural-s viel zu früh (im 19. Jahrhundert) vorkommt, um aus dem Englischen stammen zu können, vor allem aber zunächst gar nicht bei Fremdwörtern, sondern bei Eigennamen auftritt, später auch bei Kurzwörtern, Onomatopoetika und Konversionen (siehe Abb. 6). Die Etappen der Ausbreitung des Plural-s ähneln also denen des Apostrophs (zur nativen Genese des s-Plurals siehe Abschnitt 2.2).

$$
\begin{aligned}
& \text { FamN }>\text { RufN }>\text { alle Namen }>\text { Kurzwörter, Onomatopoetika, } \\
& \text { Konversionen, Fremdwörter } \\
& \text { Müllers Peters Freiburgs Loks, WCs; Uhus, Ohs; Lebewohls, } \\
& \text { Schulrens Hannas Deutschlands Warums; Sophas, Commas; }
\end{aligned}
$$

Abb. 6: Diachrone Ausbreitung des s-Plurals im Deutschen (nach Nübling/Schmuck 2010, S. 163) 
Dass der spätere Kontakt mit Fremdsprachen eine Verstärkung des s-Plurals bewirkt hat, ist nicht auszuschließen (senkrechter Pfeil in Abb. 6), doch sind sie nicht die Quelle des deutschen $s$-Plurals. Bezeichnenderweise findet morphologische Integration durch die Annahme frequenterer Pluralallomorphe wie -en, -e oder - $\varnothing$, die wohlgeformte phonologische Wörter generieren (Trochäen mit Reduktionssilbe), nur bei Fremdwörtern statt (die Kontos > Konten, die Lifts > Lifte, die Computers > Computer), nicht aber bei den Wörtern (links auf dem Pfeil in Abb. 6), deren Plural-s sich über native Wege (die Reanalyse des $s$-Genitivs) entwickelt hat: die Pontos $>$ *Ponten, die Schmidts $>{ }^{*}$ Schmidte, die Meyers $>*$ Meyer. Gleiches gilt für andere Namen, für Kurzwörter, Onomatopoetika, Konversionen und auch für viele Fremdwörter, z.B. die Sofas > *Sofen, die Kommas > Kommen. Auch machen Namen, Kurzwörter und Konversionen (sofern sie nicht deflektieren, siehe unten) systematischer vom Plural-s Gebrauch als Fremdwörter.

Die Vorteile des s-Plurals liegen in seiner den Wortkörper konstant haltenden Qualität: Es ist strikt additiv, er erfordert bzw. ermöglicht nicht einmal die Umlautung des Wurzelvokals, und er erzeugt keine weitere Silbe, lässt also die Wortlänge konstant. Silbische Suffixe wie -en, -e oder -er bewirken dagegen die Aufhebung einer Auslautneutralisierung (z.B. Kin $[t]-\operatorname{Kin}[d] e r$ ), nicht so -s (Patrick Süskin $[t]$ - die Süskin $[t] s$ ), manchmal Umlaut und immer die Erweiterung um eine Silbe. Nicht zufällig hat das Deutsche bei komplexen Substantiven ein schonendes Pluralallomorph ausgebildet.

Auch das Genitiv-s vereint die wortschonenden Vorteile des Plural-s und hat sich (bei komplexen Wörtern) diachron als so genannter überstabiler Marker gegenüber den historisch vorkommenden Allomorphen durchgesetzt, z.B. gegenüber -(e) $n$ (vgl. den Gen.Sg. von Maria: frühnhd. Marien > Mariens $>$ nhd. Marias). Wenn Allomorphie zugunsten nur eines Markers abgebaut wird, spricht man von paradigmatischer Deflexion.

Abschließend sei noch ein Blick auf die neueste Entwicklung geworfen, auf die syntagmatische (d.h. am Wortkörper selbst operierende) Deflexion von - bislang - v.a. Personen-, aber auch Örtlichkeitsnamen. Ein Nullflexiv schont den Wortkörper noch besser als ein „Schonflexiv“. Der Genitivbzw. Pluralausdruck wird hier der weiteren NP überantwortet. Genau dieses Stadium lässt sich im gegenwärtigen Deutschen beobachten, was sich in zahlreichen Zweifelsfällen und grammatikographischen Widersprüchen äußert (eingehend hierzu siehe Nübling 2012). Abermals erweist sich, dass die Eigennamen Vorreiter dieser Entwicklung sind, doch die bekannten weiteren Komplexitätsparameter (nicht-native Wortstrukturen, Kürzungen, Konversionen) auch schon ihre Wirkung entfalten. Nur dann, wenn ein Element der NP, meist der Artikel, den Genitiv bzw. den Plural markiert, unterbleibt bereits die Flexion: die Mutter des kleinen Peter (aber ohne Artikel: Peters Mutter); die beiden Deutschland(s). Vielzitiert sind die Leiden des jungen 
Werther, die in der 2. Auflage 1787 ohne -s, in der 1. Auflage 13 Jahre zuvor aber noch mit $-s$ geschrieben wurden. Als dieses Genitiv-s instabil zu werden begann, waren die Dativ- und Akkusativflexive gerade abgebaut worden (Karlen > Karl, Goethen > Goethe).

Was die heutige Situation betrifft, so schreibt der Zweifelsfall-Duden (Duden 2011, S. 717):

Familien-, Personen- und Vornamen mit vorangehendem Artikelwort bleiben ohne Flexionsendung. Der Kasus wird durch das Artikelwort angezeigt: die Partie des Lohengrin, der Pass des Anton Meyer, [...] die Bilder des jungen Dürer [...]. Wenn männliche Vornamen mit einem Artikel oder Attribut gebraucht werden, ist ebenfalls die unflektierte Form üblich: die Einfälle unseres kleinen Michael (selten, aber nicht falsch: unseren kleinen Michaels) [...].

Während - grob gesagt - die genitivische Deflexion bei Personennamen bereits Usus ist, schwanken die auf der Belebtheitshierarchie weiter hinten anzusiedelnden Toponyme noch stärker (des Iran(s?), des Balkan(s?)). Tabelle 1 enthält das Ergebnis einer Cosmas II-Recherche (vom 20.8.2012), W-Archiv der geschriebenen Sprache, mit den absoluten und relativen Ergebnissen (Prozente gerundet). Abgefragt wurden Toponyme von unterschiedlichem Fremdheitsgrad. Der Einfluss fremder Strukturen (nichtinitialer Akzent, Mehrfüßigkeit, keine Reduktionssilben, d.h. unbetonte Vollvokale etc.) auf die Deflexion (Nullendung) macht sich deutlich bemerkbar, während nativ(er)e Strukturen eher zum s-Genitiv tendieren. Hinzu kommt sicher auch der Faktor der Tokenfrequenz. Doch zeigt das Beispiel Europa, dass trotz häufigen Gebrauchs die strukturelle Markiertheit zu 73\% Nullflexion führt. Somit lässt sich auch synchron der Einfluss komplexer Wortstrukturen auf die (De-)Flexion nachweisen.

Auch andere markierte Wörter wie Fremd- und Kurzwörter sowie Konversionen neigen zur Deflexion, was grammatikographisch bereits berücksichtigt wird. So schreibt die Duden-Grammatik (2009, \ 314), dass bestimmte Substantivierungen ,nur die kurze Genitivendung, teilweise aber auch gar keine Endung“" nehmen: des Deutsch(s), des Ich $(s)$, des Wenn $(s)$. Ähnliches gelte für Initialkurzwörter (\315): des PC(s), des EKG $(s)$. Duden-Zweifelsfälle (2011) erwähnt auch die Endungslosigkeit bei Fremdwörtern (des Dativ, Enzian, Islam, Vitamin), auch wenn hier die s-Setzung empfohlen wird. Dieser Komplex soll hier nicht weiter vertieft werden, es soll nur deutlich werden, dass auch die gegenwärtige Deflexion des $s$-Genitivs den bekannten Pfad von den Eigennamen hin zu markierten Appellativen nimmt. Die genaue Sukzession dieser Deflexion (ob zuerst Fremd- oder Kurzwörter oder Konversionen) wird Gegenstand zukünftiger, korpusbasierter Forschung sein. 


\begin{tabular}{|l|c|c|r|c|}
\hline Name im Genitiv & \multicolumn{2}{|c|}{ Nullendung } & \multicolumn{2}{c|}{ s-Endung } \\
\hline & absolut & Prozent & absolut & Prozent \\
\hline des Orinoc|ko- & 82 & $96 \%$ & 3 & $4 \%$ \\
\hline des Mississippi- & 563 & $94 \%$ & 36 & $6 \%$ \\
\hline des Mekong- & 142 & $89 \%$ & 18 & $11 \%$ \\
\hline des Himalay|ja- & 963 & $83 \%$ & 191 & $17 \%$ \\
\hline des Jemen- & 379 & $83 \%$ & 77 & $17 \%$ \\
\hline des Kongo- & 620 & $73 \%$ & 230 & $27 \%$ \\
\hline des Europa- & 2348 & $73 \%$ & 859 & $27 \%$ \\
\hline des Irak- & 4227 & $68 \%$ & 1997 & $32 \%$ \\
\hline des Iran- & 2497 & $65 \%$ & 1370 & $35 \%$ \\
\hline des Sudan- & 449 & $63 \%$ & 264 & $37 \%$ \\
\hline des Engadin- & 334 & $48 \%$ & 362 & $52 \%$ \\
\hline des Ural- & 139 & $42 \%$ & 195 & $58 \%$ \\
\hline des Hunsrück- & 1236 & $35 \%$ & 2251 & $65 \%$ \\
\hline des Balkan- & 414 & $25 \%$ & 1271 & $75 \%$ \\
\hline des Schwarzwald- & 58 & $21 \%$ & 214 & $79 \%$ \\
\hline des Kaiserstuhl- & 13 & $20 \%$ & 53 & $80 \%$ \\
\hline des Rhein- & 1229 & $8 \%$ & 13787 & $92 \%$ \\
\hline
\end{tabular}

Tab. 1: Null- vs. s-Genitiv bei Toponymen (Cosmas II, 20.8.2012)

\section{Fazit}

Ausgehend von der Apostrophsetzung und ihrer diachronen Entwicklung von phonographischen zu morphographischen Funktionen konnte festgestellt werden, dass es komplexe, markierte Wortkörper sind, die der Abgrenzung flexivischer und derivationeller Suffixe bedürfen, d.h. der Distanthaltung lexikalischer von grammatischen Einheiten durch das Syngraphem Apostroph. Zentrum dieser Entwicklungen sind Eigen-, insbesondere Personennamen. Gerade hier kreuzen sich mehrere der Faktoren, die Markiertheit bewirken: Zum einen ist es ihr onymischer Status, was bedeutet, dass ein rein materieller Ausdruck ohne denotative Semantik die stabile Referenz auf ein Objekt in der Welt zu leisten hat. Zum anderen sind es nicht-native phonologische und graphematische Strukturen, die Apostrophsetzungen begünstigen. Eigennamen sind überaus häufig von Entlehnung betroffen; auBerdem tendieren sie im Gegensatz zu Appellativen, Adjektiven und Verben 
weniger dazu, integriert zu werden, d.h. sie behalten eher die fremdsprachlichen Strukturen, was wiederum ihrer monoreferenten Funktion (Notwendigkeit der Wiedererkennbarkeit) geschuldet ist. Auch Wortkürzungen (des EGK's, Joseph K.'s) bewirken häufige Apostrophsetzung, v.a. wenn sie aus Syllabogrammen bestehen. Namen werden, meist aus Gründen der Anonymisierung, oft abgekürzt. Schließlich erfahren auch nicht-genuine Nomen, d.h. Substantivierungen, häufige Apostrophsetzung. Basierend auf neueren Studien konnte nachgewiesen werden, dass der morphographische Apostroph viel älter ist als gemeinhin angenommen (bereits im 18. Jahrhundert vor Genitiv- und zuweilen auch Plural-s) und nur durch die Orthographie in den Substandard verwiesen wurde. Dort hat er indessen seine in vollem Einklang mit der graphematischen Gesamtentwicklung des Deutschen stehenden Funktionen ausgebaut, was immerhin zu seiner Duldung vor Genitiv-s nach Personennamen im Regelwerk von 1996 geführt hat.

Ein weiterer Blick auf Entstehung und Entwicklung der Flexive selbst, die der graphematischen Distanzierung durch Apostrophierung bedürfen, hat gezeigt, dass auch hier der Schutz, die Konstanthaltung des Wortkörpers die treibende Kraft war und Eigennamen diese Entwicklung angestoßen haben: Zum einen erklärt sich so die Übergeneralisierung des Genitiv-s nach Eigennamen (durch Abbau aller anderen Allomorphe) und anderen markierten Wörtern sowie die Herausbildung eines nativen Plural-s zunächst bei den Personennamen, wo $s$-Plurale früher und konsequenter erscheinen als bei Fremdwörtern. Zum anderen treten diese „Schonflexive“ gegenwärtig ihren kompletten Rückzug an, d.h. hier bahnt sich Deflexion an, sofern die NP in der Lage ist, den Genitiv bzw. Plural zu markieren. Damit dürfte der Endpunkt einer jahrhundertelangen Entwicklung erreicht sein: Komplexe Wörter, allen voran Eigennamen, erfahren die maximale Wortkörperschonung. Der Apostroph spiegelt dies auf der graphematischen Ebene.

\section{Literatur}

Augst, Gerhard (1981): Über die Schreibprinzipien. In: Zeitschrift für Phonetik, Sprachwissenschaft und Kommunikationsforschung 34, S. 734-741.

Bankhardt, Christina (2010): Tütel, Tüpflein, Oberbeistrichlein. Der Apostroph im Deutschen. (= amades 39). Mannheim.

Bergmann, Rolf (1999): Zur Herausbildung der deutschen Substantivgroßschreibung. Ergebnisse des Bamberg-Rostocker Projekts. In: Hoffmann, Walter et al. (Hg.): Das Frühneuhochdeutsche als sprachgeschichtliche Epoche. Werner Besch zum 70. Geburtstag. Frankfurt a.M., S. 59-79.

Bergmann, Rolf/Nerius, Dieter (1998): Die Entwicklung der Großschreibung im Deutschen von 1500-1700. 2 Bde. (= Germanische Bibliothek: Reihe 3, Untersuchungen; N.F. 29). Heidelberg. 
Blatz, Friedrich (1900): Neuhochdeutsche Grammatik mit Berücksichtigung der historischen Entwicklung der deutschen Sprache. 2 Bde. 3., völlig neubearb. Aufl. Hildesheim/New York.

Duden (2009): Grammatik der deutschen Gegenwartssprache. 8. Aufl. (= Duden 4). Mannheim.

Duden (2011): Richtiges und gutes Deutsch. Wörterbuch der sprachlichen Zweifelsfälle. 7., vollst. überarb. Aufl. (= Duden 9). Mannheim.

Ebert, Robert Peter et al. (1993): Frühneuhochdeutsche Grammatik. (= Sammlung kurzer Grammatiken germanischer Dialekte: A, Hauptreihe 12). Tübingen.

Eisenberg, Peter (1989): Die Grammatikalisierung der Schrift: Zum Verhältnis von silbischer und morphematischer Struktur im Deutschen. In: Mitteilungen des Deutschen Germanistenverbandes, S. 20-29.

Ewald, Petra (2006): Aus der Geschichte eines Zankapfels: Zur Entwicklung der Apostrophschreibung im Deutschen. In: Götz, Ursula/Stricker, Stefanie (Hg.): Neue Perspektiven der Sprachgeschichte. Internationales Kolloquium des Zentrums für Mittelalterstudien der Otto-Friedrich-Universität Bamberg, 11. und 12. Februar 2005. (= Germanistische Bibliothek 26). Heidelberg, S. 139-161.

Ewald, Petra/Nerius, Dieter (1999): Großschreibung der Substantive und das/daß-Differenzierung. Zur Annahme eines „grammatischen Prinzips“ in der deutschen Orthographie. In: Rostocker Beiträge zur Sprachwissenschaft 7, S. 165-186.

Gallmann, Peter (1985): Graphische Elemente der geschriebenen Sprache. Grundlagen für eine Reform der Orthographie. (= Reihe germanistische Linguistik 60). Tübingen.

Gallmann, Peter (1989): Syngrapheme an und in Wortformen. Bindestrich und Apostroph im Deutschen. In: Eisenberg, Peter/Günther, Hartmut (Hg.): Schriftsystem und Orthographie. (= Reihe Germanistische Linguistik 97). Tübingen, S. 85-110.

Grimm, Jacob (1864 [1847]): Ueber das pedantische in der deutschen sprache. In: Grimm, Jacob (Hg.): Kleinere Schriften. Bd. 1.: Reden und Abhandlungen. Berlin, S. 327-373.

Heyse, Karl Wilhelm Ludwig (1849): Theoretisch-praktische deutsche Grammatik oder Lehrbuch der deutschen Sprache, nebst einer kurzen Geschichte derselben. Bd. 2: Dr. J. C. A. Heyse's ausführliches Lehrbuch der deutschen Sprache. 5., völlig umgearb. u. sehr verm. Ausg. Hannover.

Klein, Wolf Peter (2002): Der Apostroph in der deutschen Gegenwartssprache. Logographische Gebrauchserweiterungen auf phonographischer Basis. In: Zeitschrift für Germanistische Linguistik 30, S. 169-197.

Meisenburg, Trudel (1998): Zur Typologie von Alphabetschriftsystemen anhand des Parameters der Tiefe. In: Linguistische Berichte 34, S. 43-64.

Nerius, Dieter (2007): Deutsche Orthographie. 4., neu bearb. Aufl. Hildesheim.

Nübling, Damaris (2012): Auf dem Wege zu Nicht-Flektierbaren: Die Deflexion der deutschen Eigennamen diachron und synchron. In: Rothstein, Björn (Hg.): Nichtflektierende Wortarten. (= Linguistik - Impulse \& Tendenzen 47). Berlin/New York, S. 224-246. 
Nübling, Damaris/Schmuck, Mirjam (2010): Die Entstehung des s-Plurals bei Eigennamen als Reanalyse vom Kasus- zum Numerusmarker. Evidenzen aus der deutschen und niederländischen Dialektologie. In: Zeitschrift für Dialektologie und Linguistik 77, S. 145-182.

Nübling, Damaris et al. (2010): Historische Sprachwissenschaft des Deutschen. Eine Einführung in die Prinzipien des Sprachwandels. 3., überarb. Aufl. Tübingen.

Ruge, Nikolaus (2004): Aufkommen und Durchsetzung morphembezogener Schreibungen im Deutschen 1500-1770. (= Germanistische Bibliothek 19). Heidelberg.

Sanders, Daniel (1873): Vorschläge zur Feststellung einer einheitlichen Rechtschreibung für Alldeutschland. Berlin.

Scherer, Carmen (2010): Das Deutsche und die dräuenden Apostrophe. Zur Verbreitung von 's im Gegenwartsdeutschen. In: Zeitschrift für Germanistische Linguistik 38 , S. 1-24.

Scherer, Carmen (2013): Kalb's Leber und Dienstag's Schnitzeltag. Zur funktionalen Ausdifferenzierung des Apostrophs im Deutschen. In: Zeitschrift für Sprachwissenschaft 32, S. 75-112.

Schmuck, Mirjam (2009): Personennamen als Quelle der Grammatikalisierung. Der ingDiminutiv in Mecklenburg-Vorpommern. In: Beiträge zur Namenforschung 44, S. 35-65.

Schmuck, Mirjam (2011): Vom Genitiv- zum Pluralmarker: Der $s$-Plural im Spiegel der Familiennamengeographie. In: Heuser, Rita/Nübling, Damaris/Schmuck, Mirjam (Hg.): Familiennamengeographie. Ergebnisse und Perspektiven europäischer Forschung. Berlin/New York, S. 285-304.

Szczepaniak, Renata (2010): Während des Flug(e)s/des Ausflug(e)s? German Short and Long Genitive Endings between Norm and Variation. In: Lenz, Alexandra/Plewnia, Albrecht (Hg.): Grammar Between Norm and Variation. (= VarioLingua 40). Frankfurt a.M. u.a., S. 103-126.

Szczepaniak, Renata (2011): Gemeinsame Entwicklungspfade in Spracherwerb und Sprachwandel? Kognitive Grundlagen der onto- und historiogenetischen Entwicklung der satzinternen Großschreibung. In: Köpcke, Klaus-Michael/Ziegler, Arne (Hg.): Grammatik - Lehren, Lernen, Verstehen. Zugänge zur Grammatik des Gegenwartsdeutschen. (= Reihe Germanistische Linguistik 293). Berlin/Boston, S. 341-359.

Wegera, Klaus-Peter (1996): Zur Geschichte der Adjektivgroßschreibung im Deutschen: Entwicklung und Motive. In: Zeitschrift für deutsche Philologie 115, S. 382-392.

Wustmann, Gustav (1891): Allerhand Sprachdummheiten. Kleine deutsche Grammatik des Zweifelhaften, des Falschen und des Häßlichen. Ein Hilfsbuch für alle, die sich öffentlich der deutschen Sprache bedienen. Leipzig. 
